\title{
Mulheres sem fronteiras? Uma análise da participação das mulheres no Programa Ciência sem Fronteiras da Unicamp: motivações, desafios e impactos na trajetória profissional*
}

\author{
Rebeca Buzzo Feltrin** \\ Janaina Oliveira Pamplona da Costa** \\ Léa Velho****
}

\begin{abstract}
Resumo
Este artigo apresenta como objeto de estudo o programa de mobilidade estudantil Ciência Sem Fronteiras (CsF), particularmente no contexto da Universidade Estadual de Campinas (Unicamp), buscando elucidar quem são as "mulheres sem fronteiras" da universidade e quais fatores explicativos indicam a superação dessas fronteiras. Os resultados da pesquisa indicam que as mulheres apresentam participação maior no $\mathrm{CsF}$ do que no alunado do curso em que estão matriculadas, e que o capital cultural das bolsistas do CsF da Unicamp pode ser um fator explicativo para a transposição das fronteiras no que se refere à participação em programas de mobilidade internacional.
\end{abstract}

Palavras-chave: Mobilidade Internacional, Programa Ciência sem Fronteiras, Unicamp, Mulheres, Inclusão de Gênero e Capital Cultural.

* Recebido em 30 de março de 2016, aceito em 23 de agosto de 2016.

** Pesquisadora colaboradora do Departamento de Política Científica e Tecnológica da Unicamp, Campinas, SP, Brasil; pós-doutoranda da Fundação Oswaldo Cruz, Rio de Janeiro, RJ, Brasil. rebecafeltrin@gmail.com

*** Professora do Departamento de Política Científica e Tecnológica da Unicamp, Campinas, SP, Brasil. jpamplona@ige.unicamp.br

**** Professora titular do Departamento de Política Científica e Tecnológica da Unicamp, Campinas, SP, Brasil. velho@ige.unicamp.br 
Women without Borders? A Study on The Participation of Women in Unicamp's 'Science Without Borders' Programme: Motivations, Challenges and Impacts on Professional Trajectory

\begin{abstract}
This study investigates the Brazilian international mobility 'Science without Borders' (SwB) Programme, in particular the case of Unicamp (Brazil). The article addresses how women are performing in the SwB Programme when compared to men, and which factors may explain such performance. The results show that Unicamp female students have higher participation than male students in the SwB compared to their paticipation at Unicamp courses, and suggest that cultural capital might be an explaining factor for women supersede barriers to participate in international mobility programmes.
\end{abstract}

Keywords: International Mobility, Science without Borders (Brazil), Unicamp, Women, Gender Inclusion and Cultural Capital. 


\section{Introdução ${ }^{1}$}

A internacionalização está, com destaque, na pauta da Política de Ensino Superior e da Política Científica, Tecnológica e de Inovação (PCTI) da maioria dos países industrializados e de industrialização recente. Os documentos oficiais e os programas das agências financiadoras de pesquisa e desenvolvimento (P\&D) dos mais variados países revelam que todos eles incluem programas e ações voltadas para a internacionalização do ensino superior e das atividades de pesquisa $e$ inovação, tais como: estímulo à mobilidade de estudantes e pesquisadores, colaboração física e virtual, contratos de pesquisa supranacionais, participação em organizações internacionais de pesquisa, coordenação $e$ planejamento conjunto de atividades em $\mathrm{CTI} .{ }^{2}$ Entre tais ações e programas, aqueles voltados ao estímulo à mobilidade de pessoas entre países têm sido particularmente enfatizados ${ }^{3}$, com base no argumento de que "os fluxos de conhecimento e a transferência de tecnologia são, primariamente, fenômenos incorporados em pessoas" (Mahroum, 2000:23).

1 Agradecemos a inestimável contribuição dos funcionários Mariana Pereira e Laura Ward da VRERI/Unicamp; e Silvio Souza e Orlando Furlan da DAC/Unicamp, especialmente quanto à disponibilização das informações sobre os participantes do programa, sem as quais esta pesquisa não teria sido possível.

2 Reunião realizada em Bruxelas, em 2008, confirmou que em todos os países da ERA (European Research Area), nos EUA, Austrália e Canadá, e também naqueles países referidos como BRIC (Brasil, Rússia, Índia, China) a atenção da PCTI para atividades que visam à internacionalização da $\mathrm{P} \& \mathrm{D}$ têm crescido rapidamente. Apresentações e relatórios com os mais importantes pontos discutidos naquela reunião podem ser encontrados em: www.technopolisgroup.com/intdrivers/programme.html.

3 A Comunidade Europeia, por exemplo, mantém pelo menos dois programas de mobilidade internacional que já atendeu milhares de estudantes: o Erasmus, para estudantes de graduação, e o Training and Mobility Researchers Scheme (TMR) para estudantes de pós-graduação e pós-doutores. Além de programas nacionais e supranacionais, universidades da maioria dos países promovem programas de mobilidade por meio de convênios com seus pares em outros países. 
A racionalidade do foco na mobilidade internacional é que essa é uma estratégia privilegiada para desenvolver as competências necessárias para a inserção de profissionais qualificados nas redes internacionais de produção de conhecimento (Bozeman; Corley, 2004; Wagner, 2004; Woolley et al., 2008; The Royal Society, 2011). Tais habilidades incluem a familiaridade com outras culturas, a aquisição de uma perspectiva cosmopolita, o despertar para temas globais, o aprendizado de idiomas que, argumenta-se, têm o potencial de se tornarem vantagens competitivas em termos pessoais $e$, indiretamente, nacionais (Brooks; Waters, 2011). Assim, acredita-se que é fundamental para países que querem se tornar sociedades do conhecimento e ser economicamente competitivos formar recursos humanos em quantidade e qualidade e que incluam, nesse processo, apoio à circulação internacional de seus talentos.

O Brasil também "embarcou" nesse processo de internacionalização. Vários analistas vinham consistentemente apontando problemas no isolamento da produção de conhecimento no país e na falta de articulação com a comunidade internacional (Velho, 2001, 2008; Velloso, 2002; Balbachevsky, 2006, 2009; Castro, 2007; Schwartzman, 2009). A resposta a esse diagnóstico foi uma decisão do governo, em 2011, de criar o Programa Ciência sem Fronteiras ( $\mathrm{CsF})$, com a justificativa de que "o Brasil precisa de mão de obra qualificada para garantir o próximo ciclo de desenvolvimento" 4 . Em linhas gerais, esse é um programa que, com a concessão de cerca de 100.000 bolsas de estudo no exterior no período de 4 anos,

busca promover a consolidação, expansão e
internacionalização da ciência e tecnologia, da inovação e
da competitividade brasileira, por meio do intercâmbio de
alunos de graduação e de pós-graduação e da mobilidade
internacional (Velho, 2011).

${ }^{4}$ Depoimento da presidenta Dilma Roussef, extraído do Uol Notícias http://noticias.uol.com.br/politica/2011/04/29, último acesso no dia 23 de março de 2016. 
Trata-se, portanto, de um ambicioso programa de internacionalização da produção de conhecimento brasileira, que usa como instrumento central a mobilidade de profissionais $e$ pesquisadores em formação ou formados.

O programa começou a funcionar, de forma modesta, no mesmo ano de sua criação, tomou força em 2012 e tem se mantido até 2016. Nesse período foram concedidas 92.880 bolsas em diferentes modalidades (graduação sanduíche no exterior; doutorado sanduíche no exterior; mestrado, doutorado e pósdoutorado no exterior; atração de jovens talentos, e pesquisadores visitantes). Dado o grande número de pessoas atendidas, a questão de partida deste artigo é: qual tem sido a participação de mulheres no CsF? Elas têm sido contempladas de maneira proporcional à sua presença nas universidades e carreiras privilegiadas pelo programa?

A expectativa dos resultados a partir da coleta de dados era que as mulheres tivessem baixa participação no $\mathrm{CsF}$ por duas razões principais. A primeira é que o CsF estabeleceu áreas prioritárias para concessão de bolsas que correspondem, grosso modo, a áreas tradicionalmente dominadas por homens, como as engenharias, computação e as ciências exatas e excluiu áreas mais feminizadas como as ciências sociais e humanidades. Com exceção das áreas biológicas e de saúde que, como se sabe, contam hoje com presença igualitária ou até majoritária de mulheres, as demais áreas prioritárias no $\mathrm{CsF}^{5}$ são tipicamente

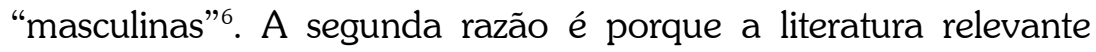
sistematicamente aponta que as mulheres são menos "móveis" que os homens - migram menos por razões de carreira e, quando

${ }^{5}$ As áreas prioritárias do $\mathrm{CsF}$ podem ser vistas no site do programa http://www.cienciasemfronteiras.gov.br e encontram-se também listadas do Quadro 2 deste artigo.

${ }^{6}$ A sub-representação das mulheres nas ciências exatas (particularmente na física), na computação e nas engenharias é documentada em tantas bases de dados e em tantos artigos para os mais diferentes países que já se tornou até conhecimento de senso comum. 
o fazem, frequentemente é para acomodar uma decisão profissional do parceiro (Clark; Withers, 2002; Ackers, 2004).

Entretanto, quando olhamos os dados do programa, vimos que nossa expectativa não se confirmava. De fato, a participação das mulheres no CsF é expressiva - cerca de $44 \%$ do total de bolsas concedidas. ${ }^{7}$ Mais surpreendente ainda, esses dados também revelam, ao contrário do que a literatura aponta de forma consistente, a ausência do que se convencionou chamar de "leaky pipeline" (conduto tubular com vazamento) de mulheres na carreira científica e tecnológica. De acordo com esse conceito, a participação de mulheres na ciência e nas engenharias decresce sensivelmente conforme elas avançam na carreira acadêmica desde a escolha do curso de graduação, passando pelo mestrado, doutorado, pós-doutorado e nos diferentes níveis como professora/pesquisadora (Blickenstaff, 2005; Etzkowitz et al, 2000; Hanson, 1996). Essas questões, usando os dados oficiais agregados do CsF, serão discutidas na seção 3 deste artigo.

A constatação da presença significativa de mulheres em todas as modalidades de bolsas de formação no $\mathrm{CsF}$ é particularmente intrigante. Afinal, quem são essas mulheres que vão para as áreas "masculinas" e buscam mobilidade internacional? Que características, que motivações as tornam "mulheres sem fronteiras" durante sua formação acadêmica? Elas têm as mesmas motivações que os homens, percebem as mesmas barreiras, respondem aos mesmos incentivos? São tão "livres" quanto eles para migrar temporariamente?

Essas perguntas não poderiam ser respondidas pelos dados agregados do programa, que, provavelmente, escondem diferenças regionais, institucionais e de áreas de conhecimento. Por isso, concentramos nosso estudo na Universidade Estadual de Campinas (Unicamp). Foi possível conseguir dados quantitativos da concessão apenas de bolsas de graduação sanduíche por curso, identificando gênero e classe social dos beneficiários. Apesar de apresentar certas limitações, a seleção da variável de origem

${ }^{7}$ http://www.cienciasemfronteiras.gov.br/web/csf/painel-de-controle 
escolar no ensino médio (escola pública ou escola privada) como indicador do nível socioeconômico dos alunos se mostrou uma alternativa confiável quando comparado a outras variáveis que tradicionalmente compõem esse indicador, como a renda familiar. A informação sobre a renda familiar dos alunos não está disponível integralmente na base de dados da universidade, já que tal informação é solicitada apenas no formulário de candidatura ao vestibular (não sendo uma informação confiável, completa, tampouco atualizada para o momento em que os alunos se inscrevem no $\mathrm{CsF}$ ). Por outro lado, a informação sobre a procedência escolar do aluno é o que garante sua pontuação extra no vestibular da Unicamp, além de ser confirmada pela instituição no momento da matrícula. De acordo com Jannuzzi (2002), a confiabilidade e a disponibilidade dos dados são ativos importantes e que devem ser considerados na escolha dos dados que irão compor o indicador socioeconômico para a análise de fenômenos sociais. Da mesma forma, a carência de informações na universidade relativas a identidade de gênero ou sexualidade dos estudantes nos levou a assumir a variável sexo, disponível no cadastro dos alunos, como indicador de gênero.

Além disso, aplicamos um questionário aos bolsistas, que incluiu várias questões abertas e, assim, obtivemos dados qualitativos esclarecedores para responder às questões que nos colocamos. Os procedimentos metodológicos empregados encontram-se descritos na seção que se segue a essa introdução (seção 1).

Os dados obtidos são organizados e analisados da seguinte maneira: a seção 2 apresenta a participação das mulheres no CsF para o nível agregado; a seção 3 apresenta o programa CsF na Unicamp com destaque para a participação das mulheres por curso. $\mathrm{O}$ perfil das mulheres participantes em termos de idade $e$ origem socioeconômica está descrito na seção 4. A seção 5 apresenta, a partir da análise dos questionários, a experiência vivenciada pelas participantes - mulheres sempre comparando com homens - em termos de motivação para o programa, impactos, expectativas de futuro. 
Em suma, este artigo discute a participação das mulheres no CsF em geral e na Unicamp em particular, com foco nas bolsas de graduação sanduíche. Busca traçar o perfil das beneficiárias $e$ identificar os motivos que as levaram a se engajar no CsF $e$ possíveis impactos dessa experiência na vida pessoal e na carreira dessas mulheres. Essas questões são sempre analisadas tendo como contraponto os homens que também participaram do CsF na Unicamp.

\section{Metodologia}

A metodologia desenvolvida nesta pesquisa contemplou a coleta de dados primários e secundários, em adição a uma breve revisão da literatura sobre programas de mobilidade internacional em diversas áreas do conhecimento. A coleta de dados primários envolveu as seguintes atividades: i) um survey eletrônico enviado aos alunos da Unicamp; ii) uso de dados disponibilizados pela Vice-Reitoria Executiva de Relações Internacionais (VRERI), que administra o CsF da Unicamp; iii) uso de dados disponibilizados pela Diretoria Acadêmica (DAC) da Unicamp, e iv) entrevista junto à representante da VRERI/Unicamp na administração do Programa CsF para essa universidade.

A coleta de dados tipo survey utilizou questionário eletrônico estruturado semiaberto, isto é, incluiu perguntas de múltipla escolha e abertas, sendo os respondentes alunos de graduação da Unicamp que foram bolsistas pelo $\mathrm{CsF}$ no período de 2012 a 2106; a participação na pesquisa foi voluntária. O objetivo do questionário foi adquirir respostas adicionais às disponibilizadas pela base de dados da universidade, assim como obter respostas qualitativas sobre as experiências dos bolsistas do Programa CsF com relação aos temas mencionados, de forma a esclarecer possíveis padrões na participação dos bolsistas do programa.

O questionário foi enviado a todos os bolsistas e ex-bolsistas do $\mathrm{CsF}$ que ainda se encontravam matriculados na Unicamp nos meses de fevereiro e março de 2016 (coleta tipo censo), isto é, 
1255 alunos da graduação (180 bolsistas já haviam concluído a graduação no momento da coleta de dados, ou abandonado a universidade). $\mathrm{O}$ envio do questionário a alunos matriculados é justificado em função da lista de endereços eletrônicos para qual o questionário foi enviado ser composta integralmente por endereços eletrônicos institucionais dos bolsistas do programa. $\mathrm{O}$ questionário utilizou a plataforma do Google Form, na qual as respostas foram automaticamente direcionadas para uma base de dados em que a identidade dos respondentes foi totalmente preservada. Obtiveram-se 204 respostas dos questionários, sendo que duas respostas foram descartadas por motivo de repetição de respondentes e dados incompletos das respostas. Dessa forma, trabalhou-se com um número total de 202 respostas, representando $16.1 \%$ dos bolsistas CsF da Unicamp. Ressalte-se que a amostra tende a ser indicativa e não exaustiva do universo de bolsistas (i.e., não representativa estatisticamente), pois tratouse de uma coleta tipo censo com taxa de resposta relativa. ${ }^{8} \mathrm{O}$ questionário abordou os seguintes temas: dados pessoais, mobilidade internacional anterior ao ingresso no CsF, escolha de universidade e país de destino, desafios e oportunidades pessoais para participação no programa.

Os dados disponibilizados pela VRERI e pela DAC referem-se ao Programa $\mathrm{CsF}$ administrado pela Unicamp para o período de 2012 a 2016, e inserem as seguintes variáveis: curso (número e nome), nível do curso (graduação, pós-graduação), área do conhecimento, sexo dos beneficiários, data de ingresso na Unicamp, data de egresso da Unicamp (quando o caso), programa de mobilidade do beneficiário, tipo de bolsa obtido, período de

8 Cabe comentar que a coleta tipo censo com $16,1 \%$ de taxa de resposta pode apresentar um viés de autosseleção dos respondentes, em que a aleatoriedade pode não vir a ser respeitada. Assim, não se faz possível garantir que a amostra coletada seja representativa do universo mais amplo de estudantes da Unicamp que foram contemplados pelo Programa CsF. Entretanto, ainda que se reconheça as restrições apontadas, a combinação de métodos quantitativos e qualitativos auxiliou na análise dos resultados e respostas às perguntas de pesquisa propostas. 
intercâmbio, universidade de destino, país de destino, cidade $e$ estado de nascimento do bolsista, e natureza da escola cursada no ensino médio (a saber, pública, ou privada).

Adicionalmente aos dados coletados sobre o $\mathrm{CsF}$, obteve-se informação sobre o número de alunos matriculados por curso na Unicamp e a proporção de homens e mulheres em cada curso. Os dados coletados sobre o $\mathrm{CsF}$ mostram que 1435 alunos da Unicamp foram beneficiados por esse programa no período referido, configurando-se como a população total que subsidiará a análise dos dados na seção 3 deste artigo. A entrevista junto à representante da VRERI Unicamp na administração do Programa $\mathrm{CsF}$ foi presencial e utilizou questionário aberto. O principal objetivo da entrevista foi entender como a universidade se estruturou para atender ao Programa $\mathrm{CsF}$ frente aos demais programas de cooperação internacional para mobilidade de seu corpo docente e discente, assim como obter informações sobre as experiências dos alunos da Unicamp no CsF.

A coleta de dados secundários refere-se aos dados disponibilizados no Painel de Controle da Plataforma Eletrônica do Programa Ciência sem Fronteiras ${ }^{9}$ para as seguintes variáveis: i) total de bolsas implementadas no país; ii) total de bolsas implementadas por modalidade, a saber, pós-doutorado no exterior, doutorado no exterior, doutorado sanduíche no exterior, mestrado no exterior, graduação sanduíche no exterior, pesquisador visitante especial, e atração de jovens talentos; iii) distribuição de bolsas de graduação por região do Brasil e unidade da federação e instituição de origem do bolsista; iv) distribuição de bolsas implementadas por áreas prioritárias, tanto no que se refere ao nível de formação (graduação, pós-graduação e outros), quanto por modalidade; v) distribuição das bolsas implementadas por país de destino; $e$ vi) as variáveis anteriores discriminadas por gênero, a saber, feminino e masculino.

9 Ver http://www.cienciasemfronteiras.gov.br/web/csf/painel-de-controle. 


\section{Participação das mulheres no Ciência sem Fronteiras}

O programa CsF concedeu, desde sua criação em 2011 até o primeiro trimestre de 2016, 92.880 bolsas em todas as modalidades - de graduação sanduíche a pós-doutorado, bolsas de atração a jovens talentos (pesquisadores brasileiros que se encontravam trabalhando no exterior e que se dispuseram a voltar ao Brasil), e bolsas para professores visitantes especiais (estrangeiros ou brasileiros radicados no exterior que se comprometem a contribuir para a pesquisa e a formação de novos pesquisadores no Brasil, passando pelo menos três meses por ano no país).

Aproximadamente $80 \%$ das bolsas do CsF correspondem à modalidade de graduação sanduíche (73.354), conforme se constata no Quadro 1. As mulheres receberam 42,7\% dessas bolsas (31.393 bolsas). A primeira reação a esse dado é afirmar que as mulheres receberam menos bolsas e que, portanto, a alocação dessas bolsas privilegiou os homens. Essa impressão é reforçada quando se compara a participação de mulheres no $\mathrm{CsF} e$ no ERAMUS, o programa de mobilidade de estudantes de graduação implementado pela Comunidade Europeia, em que as mulheres foram beneficiárias de 57\% das bolsas no período de 1987 a 1996 (Teichler; Maiworm, 1997). Entretanto, as áreas de conhecimento atendidas pelo CsF e pelo ERASMUS diferem significativamente.

Quadro 1. Participação de Mulheres por Modalidade de Bolsa, Brasil 2012-2016

\begin{tabular}{|l|r|r|r|}
\hline \multirow{2}{*}{ Modalidade de Bolsa } & \multirow{2}{*}{ Total } & \multicolumn{2}{c|}{ Mulheres } \\
\cline { 3 - 4 } & & Número & \multicolumn{1}{c|}{$\%$} \\
\hline Graduação Sanduíche & 73.354 & 31.393 & 42,7 \\
\hline Mestrado & 558 & 169 & 30,2 \\
\hline Doutorado & 3.353 & 1.459 & 43,5 \\
\hline Doutorado Sanduíche & 9.658 & 5.193 & 53,7 \\
\hline Pós-doutorado & 4.652 & 2.036 & 43,7 \\
\hline Atração Jovens Talentos & 504 & 198 & 39,2 \\
\hline Professor Visitante especial & 775 & 40 & 5,1 \\
\hline Total & 92.880 & 40.488 & 43,5 \\
\hline
\end{tabular}

Fonte: Painel de Controle, Ciência sem Fronteiras. 
O programa ERASMUS atende estudantes de todas as áreas do conhecimento. De fato, cerca de $20 \%$ dos estudantes desse programa eram da área de administração, $e$ as áreas de direito, sociologia e línguas receberam, cada uma delas, aproximadamente 10\% das bolsas (Teichler; Maiworm, 1997:37). Portanto, apenas essas quatro áreas, em que a participação das mulheres é majoritária, corresponderam a $50 \%$ das bolsas do programa e certamente pressionaram para cima a participação de mulheres no total de bolsas distribuídas. A composição de áreas prioritárias do CsF é completamente diferente, como se pode ver no Quadro 2: as engenharias e outras áreas tecnológicas receberam praticamente metade das bolsas do $\mathrm{CsF} e$ as ciências sociais e humanidades não foram incluídas no programa. $\mathrm{Na}$ medida em que homens e mulheres habitam de forma diferenciada as áreas de conhecimento, é de se esperar que, para ser equitativo, o programa de mobilidade deveria atender cada sexo de modo proporcional à presença de um e de outro no alunado de graduação.

Quadro 2. Participação de mulheres por área prioritária

\begin{tabular}{|l|r|r|r|}
\hline \multirow{2}{*}{ Área Prioritária } & \multirow{2}{*}{${ }^{10}$} & \multirow{2}{*}{ Total } & \multicolumn{2}{c|}{ Mulheres } \\
\cline { 3 - 4 } & & \multicolumn{1}{c|}{ Número } & \multicolumn{1}{c|}{$\%$} \\
\hline $\begin{array}{l}\text { Engenharias e demais áreas } \\
\text { tecnológicas }\end{array}$ & 41.594 & 14.002 & 33,6 \\
\hline $\begin{array}{l}\text { Biologia, Ciências Biomédicas e } \\
\text { da saúde }\end{array}$ & 16.076 & 10.009 & 62,2 \\
\hline Indústria criativa & 8.061 & 5.237 & 64,9 \\
\hline Ciências exatas e da terra & 7.361 & 2.992 & 40,6 \\
\hline $\begin{array}{l}\text { Computação e Tecnologias da } \\
\text { Informação }\end{array}$ & 5.694 & 973 & 17,0 \\
\hline Produção agrícola sustentável & 3.197 & 1.575 & 49,2 \\
\hline Biotecnologia & 2.039 & 1.199 & 58,8 \\
\hline
\end{tabular}

\footnotetext{
${ }^{10} \mathrm{O}$ total de bolsas por área prioritária neste quadro não inclui aquelas que não identificaram a área e, por essa razão, difere do total do Quadro 1.
} 


\begin{tabular}{|l|r|r|r|}
\hline Fármacos & 1.879 & 1.301 & 69,2 \\
\hline Biodiversidade e bioprospeção & 1.342 & 703 & 52,3 \\
\hline Energias renováveis & 1.000 & 423 & 42,3 \\
\hline Ciências do Mar & 813 & 485 & 59,6 \\
\hline $\begin{array}{l}\text { Nanotecnologia e Novos } \\
\text { Materiais }\end{array}$ & 697 & 282 & 40,4 \\
\hline Petróleo, gás e carvão mineral & 678 & 265 & 39,0 \\
\hline $\begin{array}{l}\text { Novas tecnologias de engenharia } \\
\text { construtiva }\end{array}$ & 566 & 302 & 53,3 \\
\hline Tecnologia aeroespacial & 431 & 0 & 0 \\
\hline $\begin{array}{l}\text { Tecnologias de mitigação de } \\
\text { desastres }\end{array}$ & 260 & 0 & 0 \\
\hline Formação de tecnólogos & 183 & 0 & 0 \\
\hline Tecnologia mineral & 136 & 0 & 0 \\
\hline Total & 92.007 & 39.748 & - \\
\hline
\end{tabular}

Fonte: Painel de Controle, Ciência sem Fronteiras.

Dados oficiais informam que nas engenharias, por exemplo, as mulheres correspondem a $33,6 \%$ das matrículas nas universidades públicas brasileiras (INEP, 2013, Tabela 1.8) ${ }^{11}$. E, de acordo com o Quadro 2, elas receberam 33,6\% das bolsas do CsF alocadas para as engenharias. Portanto, ainda que tenham recebido menor número de bolsas que os homens, as mulheres foram atendidas na mesma proporção em que participam do alunado das engenharias. Dados do ERASMUS indicam uma situação análoga: as mulheres europeias receberam $38 \%$ das bolsas nas engenharias, que, no caso, "resultou numa representação mais alta do que a participação delas no alunado destas áreas" (Teichler; Maiworm, 1997:39).

Em várias áreas prioritárias do $\mathrm{CsF}$ também se nota uma participação expressiva das mulheres (Quadro 2), incluindo a produção agrícola (quase $50 \%$ de bolsistas mulheres) e as ciências exatas e da terra $(40,6 \%)$, áreas essas em que a presença de

\footnotetext{
${ }^{11} \mathrm{~A}$ definição de áreas usada pelo INEP não corresponde exatamente àquela adotada pelo CsF, o torna a comparação menos rigorosa do que o desejado. Entretanto, para os fins da análise que se pretende aqui, a aproximação é válida.
} 
mulheres no alunado de graduação é tradicionalmente mais baixa que a dos homens, conforme dados do INEP - 47\% e 34,8\% respectivamente (INEP, 2013, Tabela 1.8). Nas áreas de biotecnologia, fármacos, biodiversidade e bioprospeção e ciências do mar, as mulheres receberam mais, ou bem mais, de metade do número de bolsas. $\mathrm{O}$ caso da computação e tecnologias da informação vale ser mencionado porque, segundo estudos nacionais, a participação de mulheres no alunado de graduação nessa área não apenas é a mais baixa de todas (menor que 20\%), como vem decrescendo na última década, atingindo entre 5 e 10\% apenas (Lima, 2013). Portanto, até mesmo sobre essa área, que mostra a menor participação relativa de mulheres no CsF (17\%), não se pode afirmar que as mulheres foram mal atendidas pelas bolsas na medida em que elas o foram na mesma (ou maior) proporção em que participam do alunado. Finalmente, nas últimas quatro áreas prioritárias que constam do Quadro 2, nota-se uma total ausência de bolsistas mulheres. Em nenhum desses casos é possível fazer uma comparação válida com a composição de sexos no alunado de graduação porque essas áreas prioritárias não constam como tais na composição do alunado nem nos dados do INEP nem daqueles do IBGE. Mas, fica a indagação das razões para essa ausência e de quais seriam as características dessas áreas que não atraem mulheres.

Em suma, o que se pode dizer da participação de mulheres no programa $\mathrm{CsF}$ é que, ainda que elas recebam menor número de bolsas que os homens, elas estão igualmente ou mais fortemente representadas no $\mathrm{CsF}$ do que na população de alunos de graduação das áreas prioritárias do programa. Essa conclusão é reforçada pela análise dos dados do Quadro 1. Como já mencionado, as mulheres estão menos representadas conforme avançam os níveis de escolaridade e da carreira profissional, segundo um enorme número de estudos. Entretanto, esse fenômeno não se manifestou no caso do CsF. A proporção de mulheres beneficiárias de bolsas de doutorado e pós-doutorado é praticamente a mesma que recebeu bolsas de graduação. Além disso, as mulheres receberam mais de metade das bolsas de 
doutorado sanduíche do programa (53,7\%). Portanto, as mulheres não parecem estar em desvantagem no contexto do $\mathrm{CsF}$ - as desvantagens que elas ainda registram remontam aos obstáculos que elas encontraram ao longo da vida escolar e do contexto social e que as desestimulam a ingressar nas carreiras de ciências "duras" e engenharias eleitas pelo programa.

\section{O programa Ciência sem Fronteiras na Unicamp}

Em 2015, a Unicamp contava com 34.616 matrículas, sendo 18.698 alunos nos cursos de graduação e 15.918 alunos regulares na pós-graduação. ${ }^{12}$ Apesar de o número de alunos matriculados na Unicamp não ser tão expressivo quando comparado a outras universidades brasileiras, a Unicamp ocupa o quinto lugar entre as universidades que mais receberam bolsas do CsF no Brasil, e o segundo lugar no estado de São Paulo. Entre 2012 a 2015, foram concedidas 2.384 bolsas do CsF aos alunos da Unicamp em diferentes modalidades. ${ }^{13}$ Desse total, até janeiro de 2016, foram destinadas 1.435 bolsas aos alunos de graduação da universidade, sendo 618 mulheres e 817 homens (VRERI/Unicamp), que compóem o objeto de estudo deste artigo.

${ }^{12}$ Anuário Estatístico da Unicamp, 2015.

${ }^{13}$ Disponível em: http://www.cienciasemfronteiras.gov.br/web/csf/painel-decontrole. 
Quadro 3. Total de alunas por curso e total de alunas bolsistas, CsF Unicamp (2012-2015)

\begin{tabular}{|c|c|c|c|c|c|c|}
\hline \multirow{3}{*}{ Nome do Curso } & \multicolumn{3}{|c|}{ Alunas por curso $^{14}$} & \multicolumn{3}{|c|}{ Alunas CsF por curso } \\
\hline & \multirow{2}{*}{ Total } & \multicolumn{2}{|c|}{ Mulheres } & \multirow{2}{*}{ Total } & \multicolumn{2}{|c|}{ Mulheres } \\
\hline & & \# & $\%$ & & \# & $\%$ \\
\hline $\begin{array}{l}\text { Arquitetura e } \\
\text { Urbanismo }\end{array}$ & 207 & 148 & 71,5 & 48 & 40 & 83,3 \\
\hline $\begin{array}{l}\text { Ciência da } \\
\text { Computação }\end{array}$ & 301 & 37 & 12,3 & 38 & 3 & 7,9 \\
\hline Ciências Biológicas & 249 & 156 & 62,6 & 37 & 29 & 78,4 \\
\hline $\begin{array}{l}\text { Comunicação Social } \\
\text { - Midialogia }\end{array}$ & 145 & 85 & 58,6 & 13 & 11 & 84,6 \\
\hline Educação Física & 598 & 258 & 43,1 & 19 & 8 & 42,1 \\
\hline Enfermagem & 200 & 185 & 92,5 & 7 & 7 & 100 \\
\hline Engenharia Agrícola & 421 & 115 & 27,3 & 24 & 12 & 50,0 \\
\hline Engenharia Civil & 514 & 156 & 30,3 & 111 & 39 & 35,1 \\
\hline $\begin{array}{l}\text { Engenharia de } \\
\text { Alimentos }\end{array}$ & 709 & 498 & 70,2 & 143 & 117 & 81,8 \\
\hline $\begin{array}{l}\text { Engenharia de } \\
\text { Computação }\end{array}$ & 573 & 62 & 10,8 & 114 & 12 & 10,5 \\
\hline $\begin{array}{l}\text { Engenharia de } \\
\text { Controle e } \\
\text { Automação }\end{array}$ & 339 & 34 & 10,0 & 38 & 3 & 7,9 \\
\hline $\begin{array}{l}\text { Engenharia de } \\
\text { Manufatura }\end{array}$ & 357 & 107 & 30,0 & 34 & 13 & 38,2 \\
\hline $\begin{array}{l}\text { Engenharia de } \\
\text { Produção }\end{array}$ & 359 & 123 & 34,2 & 107 & 41 & 38,3 \\
\hline $\begin{array}{l}\text { Engenharia de } \\
\text { Telecomunicações }\end{array}$ & 198 & 32 & 16,2 & 5 & 0 & 0 \\
\hline Engenharia Elétrica & 654 & 81 & 12,4 & 110 & 13 & 11,8 \\
\hline Engenharia Mecânica & 876 & 100 & 11,4 & 138 & 23 & 16,7 \\
\hline Engenharia Química & 626 & 247 & 39,5 & 172 & 82 & 47,7 \\
\hline
\end{tabular}

\footnotetext{
${ }^{14}$ Os valores de "alunas por curso" se referem ao número total de alunos por curso, número absoluto de alunas sobre o alunado e percentual de alunas sobre o alunado; valores de "alunas $\mathrm{CsF}$ por curso" se referem ao número total de bolsistas do CsF por curso, número absoluto de mulheres bolsistas e percentual de alunas sobre o número total de bolsistas.
} 


\begin{tabular}{|l|c|c|c|c|c|r|} 
Estatística & 318 & 128 & 40,2 & 6 & 5 & 83,3 \\
\hline Farmácia & 226 & 181 & 80,1 & 37 & 33 & 89,2 \\
\hline Física & 259 & 52 & 20,1 & 22 & 5 & 22,7 \\
\hline Geografia & 196 & 81 & 41,3 & 7 & 4 & 57,1 \\
\hline Geologia & 154 & 64 & 41,6 & 25 & 17 & 68,0 \\
\hline $\begin{array}{l}\text { Licenciatura em } \\
\text { Ciências Biológicas }\end{array}$ & 247 & 152 & 61,5 & & & 58,3 \\
\hline Matemática & 61 & 15 & 24,6 & 6 & 3 & 50,0 \\
\hline Medicina & 711 & 400 & 56,3 & 37 & 26 & 70,3 \\
\hline Nutrição & 257 & 216 & 84,0 & 12 & 3 & 25,0 \\
\hline Odontologia & 388 & 284 & 73,2 & 4 & 4 & 100 \\
\hline Química & 394 & 193 & 49,0 & 28 & 11 & 39,3 \\
\hline Química Tecnológica & 252 & 142 & 56,3 & 8 & 4 & 50,0 \\
\hline $\begin{array}{l}\text { Superior Tecn. } \\
\text { Análise e Desenv. } \\
\text { Sistemas }\end{array}$ & 255 & 51 & 20,0 & 20 & 4 & 20,0 \\
\hline $\begin{array}{l}\text { Superior Tecn. em } \\
\text { Saneamento } \\
\text { Ambiental }\end{array}$ & 29 & 20 & 67,0 & 5 & 4 & 80,0 \\
\hline $\begin{array}{l}\text { Superior Tecn. em } \\
\text { Construção de } \\
\text { Edifícios }\end{array}$ & 217 & 100 & 46,1 & 5 & 4 & 80,0 \\
\hline Total & 11290 & 4503 & 39,8 & 1404 & 594 & 42,3 \\
\hline
\end{tabular}

Fonte: construção própria a partir de dados fornecidos pela DAC e VRERI Unicamp. ${ }^{15}$

Segundo informações da DAC/Unicamp, o número de mulheres matriculadas na graduação da Unicamp em 2016 é 8.190

\footnotetext{
${ }^{15}$ Os dados sobre alunos por curso correspondem àqueles matriculados no ano de 2016. Os cursos aqui listados são apenas aqueles que tiveram pelo menos cinco estudantes bolsistas no período de 2012 a 2015. Considerou-se que os cursos com menos de cinco alunos bolsistas do CsF poderiam distorcer a análise. A exceção foi o curso de odontologia que, apesar de ter apenas quatro estudantes bolsistas no período, foi considerado por ser um curso com muitos alunos matriculados (388), que, aparentemente, têm demonstrado pouco interesse na mobilidade internacional. Devido à exclusão dos cursos com menos de cinco bolsistas, o total de bolsistas aqui é menor do que o total da Unicamp mencionado neste artigo.
} 
(44\%), e o número de homens matriculados é 10.325 (56\%), revelando a manutenção de uma leve disparidade na participação de gêneros. Essa disparidade fica mais evidente quando se apresenta, no Quadro 3, a participação de mulheres apenas nos cursos que tiveram bolsistas do programa $\mathrm{CsF}$ - menos de $40 \%$ do alunado desses cursos são mulheres. Ou seja, a escolha das áreas prioritárias do $\mathrm{CsF}$ poderia, potencialmente, diminuir a probabilidade de participação das mulheres. Entretanto, a boa notícia é que isso não ocorreu. De fato, as mulheres foram contempladas na Unicamp com $42,3 \%$ das bolsas, mais de 2 pontos percentuais acima do que se esperaria pela participação delas no alunado elegível.

Analisando a composição do alunado de graduação da Unicamp a partir apenas dos cursos que receberam bolsas do CsF, nota-se a persistência do padrão convencional de cursos "masculinos" e cursos "femininos" (Quadro 3). As mulheres são maioria em cursos voltados ao cuidado (care $)^{16}$, como enfermagem, odontologia, nutrição, farmácia $e$, em menor proporção, até mesmo medicina. Nas áreas mais "duras" $e$ tecnológicas, a participação das mulheres se concentra na engenharia de alimentos e na engenharia química, cursos também considerados "femininos" e "permitidos" às mulheres (Lombardi, 2006).

As áreas tecnológicas "duras" ou mais "científicas", tradicionalmente consideradas como masculinas, em geral obtêm maior prestígio e reconhecimento social. As raízes desse fato estão atreladas a uma associação frequente entre os valores tidos como "científicos" e aqueles atribuídos ao domínio masculino, como objetividade, razão, controle, dominação (Keller, 1991). FaustoSterling (2001) revela que o fato de as mulheres não aprenderem ou não se dedicarem às áreas mais "duras" da ciência em nada tem a ver com suas capacidades físicas ou mentais, mas com expectativas e oportunidades diferentes ligadas a normas de

\footnotetext{
${ }^{16}$ Sobre a discussão envolvendo a ligação do trabalho feminino à área de cuidados, ver Hirata e Guimarães (2012).
} 
gênero socialmente estabelecidas que as direcionam para áreas que satisfaçam tais expectativas. Tais expectativas incluem $\mathrm{O}$ cuidado com os filhos e a adaptação da carreira das mulheres às necessidades de cuidados da família, o que faz com que a mobilidade internacional das mulheres seja mais limitada que a dos homens, particularmente, mas não apenas, quando as mulheres têm filhos (González; Vergés, 2013).

No estágio da graduação, entretanto, quando as mulheres, em geral, ainda não têm filhos, a participação delas nos programas de mobilidade internacional sofre menos limitações. $\mathrm{E}$, conforme evidenciado pelos dados do Quadro 3, elas fazem bom uso dessa oportunidade. $\mathrm{Na}$ grande maioria das áreas de conhecimento, as mulheres receberam uma proporção maior de bolsas do $\mathrm{CsF}$ do que a participação delas no alunado. Em algumas áreas, tais como engenharia agrícola e estatística, elas dobraram a presença, em termos percentuais, no CsF comparado com a presença no curso. Portanto, mais uma vez pode-se afirmar que no contexto do $\mathrm{CsF}$, pelo menos no nível das bolsas de graduação sanduíche, as mulheres da Unicamp conseguiram obter sucesso mais que proporcional à participação delas nos cursos em que estão matriculadas.

Ao analisarmos a participação feminina em outros programas de mobilidade da Unicamp, que incluem as carreiras de artes e humanidades, no mesmo período de implementação do CsF (2012-2015), percebemos que a exclusão dessas áreas teve um impacto significativo sobre a participação das mulheres no CsF, tendo em vista que as mulheres representam a maioria entre os alunos beneficiários desses outros programas de mobilidade na instituição. 
Quadro 4. Participação de alunas da Unicamp em programas de mobilidade internacional, 2012-2015

\begin{tabular}{|l|c|c|c|}
\hline $\begin{array}{c}\text { Programa de Mobilidade } \\
\text { Internacional }\end{array}$ & $\begin{array}{c}\text { Total de } \\
\text { alunos }\end{array}$ & $\begin{array}{c}\text { Número de } \\
\text { Mulheres }\end{array}$ & $\begin{array}{c}\% \\
\text { Mulheres }\end{array}$ \\
\hline CsF & 1435 & 618 & $43 \%$ \\
\hline $\begin{array}{l}\text { Outros programas de } \\
\text { mobilidade (disciplinas de } \\
\text { graduação e de pós- } \\
\text { graduação no exterior) }\end{array}$ & 367 & 224 & $61 \%$ \\
\hline $\begin{array}{l}\text { Duplo diploma (cursos de } \\
\text { engenharia) }\end{array}$ & 46 & 9 & $19 \%$ \\
\hline
\end{tabular}

Fonte: elaboração própria baseada em dados disponibilizados pela VRERI e DAC Unicamp.

Não há dúvidas de que as mulheres da Unicamp participam ativamente das oportunidades de mobilidade na graduação, sendo sua participação proporcionalmente maior que a dos homens (no caso do $\mathrm{CsF}$ ) ou mesmo numericamente superior a dos homens (no caso de outros programas de mobilidade). Por que isso ocorre? Por que essa mulheres são mais motivadas que seus colegas homens a participarem de oportunidades de mobilidade internacional? É dessas questões que tratamos nas próximas seções.

\section{Mulheres sem fronteiras: quem são as mulheres incluídas pelo CsF?}

Conforme apresentado na Introdução e na Seção 1 deste artigo, o Programa CsF tem como objetivo fomentar a mobilidade internacional em áreas prioritárias do conhecimento por meio de

\footnotetext{
${ }^{17}$ Apenas na modalidade do Duplo Diploma as mulheres apresentam uma participação menor. De acordo com a gestora institucional, o duplo diploma é um programa voltado exclusivamente às engenharias. Essa opção iniciou-se a partir de um convênio entre a Usp e a Unicamp e universidades francesas para a engenharia elétrica. Logo depois passou a incluir outras engenharias, como a engenharia mecânica, a engenharia química, etc. A gestora enfatiza que há a intenção de incluir a engenharia de alimentos, o que favoreceria o aumento da participação das mulheres nessa modalidade já que "é um curso mais feminino".
} 
concessão de bolsas, especialmente em áreas tecnológicas. Mostrou-se na Seção 3 que o número de alunas bolsistas do $\mathrm{CsF}$ pela Unicamp é mais que proporcional ao número de alunos bolsistas em vários cursos da Unicamp (ver Quadro 3). Tal evidência remete à pergunta introduzida anteriormente neste artigo: quem são as 'mulheres sem fronteiras' da Unicamp? Ou seja, por quem é formado o grupo de mulheres que consegue transpor duas "fronteiras": a primeira, fronteira de acesso às áreas mais "valorizadas" da ciência e reconhecidas por serem de grande concentração masculina; e, a segunda, fronteira de acesso às bolsas dos programas de mobilidade destinadas a um grupo majoritariamente masculino no número de alunos por curso.

A resposta à pergunta colocada acima requer um indicador de análise adicional ao de gênero, a saber, o nível socioeconômico dos alunos, aqui analisado sob a proxy de origem das escolas do ensino médio dos bolsistas do CsF da Unicamp - escolas públicas e escolas privadas.

Os dados fornecidos pela VRERI e pela DAC/Unicamp mostram que a maior parte dos bolsistas do CsF da Unicamp é de homens que vieram da escola privada, seguidos de mulheres com a mesma origem de escola no ensino médio. Bolsistas homens e mulheres com origem em escolas públicas de ensino médio ocupam as $3^{\text {a }}$ e $4^{\mathrm{a}}$ posições respectivamente (Quadro 5).

Quadro 5. Bolsistas CsF da Unicamp, amostra selecionada, gênero e origem das escolas

\begin{tabular}{|l|c|r|c|r|}
\hline \multirow{2}{*}{ Escola } & \multicolumn{2}{|c|}{ Mulher } & \multicolumn{2}{c|}{ Homem } \\
\cline { 2 - 5 } & $\#$ & $\%$ & $\#$ & \multicolumn{1}{c|}{$\%$} \\
\hline Pública & 140 & 22,65 & 232 & 27,85 \\
\hline Privada & 462 & 74,76 & 570 & 68,43 \\
\hline Exterior & 1 & 0,16 & 2 & 0,24 \\
\hline Não informado & 15 & 2,43 & 29 & 3,48 \\
\hline Total & 618 & 100 & 833 & 100 \\
\hline
\end{tabular}

Fonte: elaboração própria baseada em dados fornecidos pela VRERI $e$ DAC/Unicamp. 
Considerando a clara priorização do CsF por cursos tecnológicos, era esperado que bolsistas homens fossem os principais beneficiários do CsF em números absolutos. Entretanto, quando adicionamos à análise o indicador de nível socioeconômico, pode-se observar que a origem das escolas cursadas no ensino médio exerce influência direta sobre o indicador de gênero. Dessa forma, ainda que o universo da amostra seja majoritariamente masculino, o segundo perfil mais frequente é o da mulher de escola privada.

Segundo McCall (2005), o conceito de "interseccionalidade" revela que os marcadores sociais de diferença (como sexo/gênero, nível socioeconômico, raça/etnia, etc.) não operam isoladamente. Tais marcadores podem ou não se traduzir em desigualdades sociais. Quando combinados, esses marcadores sociais podem contribuir para a produção de novos significados, ou ainda, outros tipos de desigualdades. Assim, uma análise interseccional oferece uma visão mais ampla e sensivel para explicar os fenômenos permeados pelas complexidades sociais.

No caso dos bolsistas do CsF da Unicamp, a análise dos indicadores socioeconômicos (i.e., escolas de origem no ensino médio) em conjunto com o indicador de gênero auxilia no entendimento do grupo de mulheres sem fronteiras que participam de programas de mobilidade oferecidos por essa universidade. A inclusão de gênero propiciada por esses programas é parcial $e$ direcionada, já que beneficia um perfil muito específico de mulher: as mulheres que cursaram a maior parte do ensino médio em escola privada. Vale observar que a alunado geral da Unicamp responde, na maioria das vezes, a um perfil muito específico de universidade - com alta tradição em pesquisa, elitizada $e$ excludente desde o processo de seleção/acesso às vagas por meio do vestibular, embora tenha se investido muito nos últimos anos na criação de mecanismos para a inclusão social, como é o caso do Programa de Ação Afirmativa e Inclusão Social (PAAIS) e o Programa de Formação Interdisciplinar Superior (PROFIS).

Ainda que os dados gerais do CsF da Unicamp evidenciem um perfil específico de mulher que se beneficia do programa, faz- 
se necessário compreender melhor as intersecções entre gênero $e$ os indicadores socioeconômicos a partir da análise dos dados qualitativos sobre as experiências prévias dos bolsistas do CsF, objeto da próxima seção.

\section{Experiências no CsF: motivação para adesão ao programa, impactos e expectativas de futuro}

Conforme discutido na Seção 1 (Metodologia), este estudo realizou coleta de dados primários junto aos bolsistas do $\mathrm{CsF}$ da Unicamp por meio de questionário tipo survey. A amostra obtida com os questionários representa $16,1 \%$ dos bolsistas da Unicamp no período de 2012 a 2016. Dessa forma, os dados apresentados a partir dos questionários são indicativos do perfil e das experiências dos bolsistas, e não dados dos quais podem ser extraídas inferências estatísticas. Apesar disso, a proporção de homens e mulheres participantes do $\mathrm{CsF}$ (total e respondentes do questionário) se manteve praticamente a mesma (43\% mulheres participantes do $\mathrm{CsF}$ no geral e $42 \%$ de mulheres respondentes dos questionários). Também houve relativo equilibrio na proporção de alunos oriundos de escolas pública e privada quando comparamos o universo geral de participantes do programa com a amostra selecionada.

A amostra de respondentes é composta por 204 bolsistas, 86 mulheres e 118 homens. Segundo os questionários, a maior parte dos ex-bolsistas tinha entre 20 e 22 anos no início do intercâmbio ${ }^{18}$, e a idade média dos bolsistas no momento da coleta de dados foi predominantemente entre 23 e 25 anos de idade. ${ }^{19}$ Com relação às escolas de origem no ensino médio, entre os alunos da amostra, os resultados para homens e mulheres foi equilibrado: $27,90 \%$ das mulheres estudaram em escolas públicas no ensino médio versus $27,96 \%$ dos homens, e $72,09 \%$ das

\footnotetext{
18 Segundo dados da Pesquisa Nacional por Amostra de Domicílios (Pnad) realizada pelo Instituto Brasileiro de Geografia e Estatística (IBGE) em 2012, 24\% dos brasileiros entre 25 e 34 anos de idade ainda vivem na casa dos pais.

${ }^{19}$ Apenas 19 alunos na amostra tinham mais de 27 anos.
} 
mulheres estudaram em escolas privadas no ensino médio versus 72,03\% dos homens.

Ainda que a distribuição por gênero dos bolsistas do $\mathrm{CsF}$ da Unicamp oriundos de escolas públicas e privadas tenha sido equilibrada, buscou-se obter dados qualitativos que pudessem sugerir padrões explicativos do perfil das mulheres sem fronteiras da Unicamp, em adição à sua origem de escola privada.

Um dos objetivos do questionário foi coletar tais dados indicativos de capital cultural (Bourdieu, 1998) dos bolsistas do CsF da Unicamp. Segundo Adamuti-Trache e Andres (2008:9), que se baseiam nas contribuições de Bourdieu em seus estudos, entendese por capital cultural "atributos específicos transmitidos pela família que influenciaram as realizações profissionais de alguém". Nesse caso, o capital cultural transmitido pelos pais é essencial para a escolha das carreiras das mulheres, qual seja, áreas do conhecimento de ciências "duras". Dessa forma, levantou-se informação sobre as seguintes experiências dos bolsistas relacionadas ao capital cultural: i) se a viagem de intercâmbio pelo $\mathrm{CsF}$ tinha sido a primeira experiência de viagem ao exterior; ii) se o bolsista tinha conhecimento suficiente do idioma no momento da escolha do país/universidade no exterior; iii) se o bolsista recebeu visitas de familiares e/ou amigos brasileiros durante o período de estadia no exterior; e iv) se o bolsista havia planejado fazer um estágio no exterior durante a graduação antes de conhecer o Programa CsF. O Quadro 6 disponibiliza os dados de capital cultural dos bolsistas do CsF da Unicamp para o período investigado, considerando também as intersecções entre gênero e origem das escolas no ensino médio. 
Quadro 6. Experiências selecionadas (capital cultural), bolsistas CsF da Unicamp, amostra selecionada por gênero e origem de escola

\begin{tabular}{|c|c|c|c|c|c|}
\hline \multirow[t]{2}{*}{ Experiência } & \multirow[t]{2}{*}{ Gênero } & \multicolumn{2}{|c|}{$\begin{array}{c}\text { Escola Privada } \\
\%\end{array}$} & \multicolumn{2}{|c|}{$\begin{array}{c}\text { Escola Pública } \\
\%\end{array}$} \\
\hline & & Não & Sim & Não & Sim \\
\hline \multirow{2}{*}{$\begin{array}{l}\mathrm{CsF} 1^{\mathrm{a}} \text { viagem ao } \\
\text { exterior* }\end{array}$} & Feminino & 64,5 & 35,5 & 66,7 & 33,3 \\
\hline & Masculino & 61,2 & 38,8 & 18,2 & 81,8 \\
\hline \multirow{2}{*}{$\begin{array}{l}\text { Conhecimento prévio de } \\
\text { idioma** }\end{array}$} & Feminino & 21,0 & 79,0 & 20,8 & 79,2 \\
\hline & Masculino & 23,5 & 76,5 & 39,4 & 60,6 \\
\hline \multirow{2}{*}{$\begin{array}{l}\text { Recebeu visitas durante } \\
\text { período no exterior**** }\end{array}$} & Fem & 24,2 & 75,8 & 33,3 & 66,7 \\
\hline & Masculino & 34,1 & 65,9 & 44,5 & 55,5 \\
\hline \multirow{2}{*}{$\begin{array}{l}\text { Planejava estágio no } \\
\text { exterior antes de } \\
\text { conhecer o } \mathrm{CsF}^{* * * * *}\end{array}$} & Feminino & 56,5 & 43,5 & 54,2 & 45,8 \\
\hline & Masculino & 64,7 & 35,3 & 54,5 & 45,5 \\
\hline
\end{tabular}

Fonte: elaboração própria baseada em dados coletados em questionário tipo survey.

Nota: Estas experiências se referem às seguintes perguntas no questionário: * A viagem de intercâmbio pelo $\mathrm{CsF}$ foi sua primeira experiência internacional?; *** Você tinha conhecimento suficiente do idioma no momento da escolha do país/universidade no exterior? *** Recebeu visitas de familiares/amigos brasileiros durante o período no exterior? **** Você havia planejado fazer um estágio no exterior durante a graduação antes de conhecer o $\mathrm{CsF}$ ?

Os dados sugerem que as mulheres bolsistas do CsF da Unicamp tiveram acesso a um capital cultural mais elevado que os homens até o momento de implementação da bolsa do programa, tanto as oriundas de escolas privadas como as de escolas públicas. $\mathrm{O}$ fato de as mulheres bolsistas receberem mais visitas da família enquanto residiam fora do país e de obterem conhecimento prévio de língua estrangeira em maior proporção do que os homens, sugere que as mulheres sem fronteiras da Unicamp recebem maior acompanhamento $e$ incentivo familiar (possivelmente dos pais) em suas trajetórias escolares; mais do que os homens beneficiados pelo programa, e em especial os homens oriundos de escola 
pública. A mesma inferência pode ser feita com relação a viagens prévias ao exterior. Assim, a participação no CsF indica ser a continuidade de um processo anteriormente iniciado na aquisição de capital cultural e acaba por ser um fator complementar que consolida uma trajetória das bolsistas do CsF da Unicamp na aquisição desse atributo.

Segundo dados do ERASMUS (Teichler; Maiworm, 1997), a experiência prévia de visitas e estadas em outros países tornam as pessoas mais interessadas em participar de programas de mobilidade internacional, e pode fazer parte do capital cultural possivelmente patrocinado pela família. Ressalte-se que a coleta de dados via questionário tipo survey não criou um grupo de controle, e como não coletou dados sobre a experiência de viagens daqueles que não participaram do CsF da Unicamp, não é possivel afirmar que esse capital cultural apresentou peso na decisão das bolsistas, mas é uma possível hipótese explicativa. Respostas qualitativas no questionário contribuem para que essa hipótese seja válida, conforme relatos dos bolsistas:

[...] já tinha realizado estágio na França e gostaria de fazer um novo estágio fora em país de língua inglesa (mulher, ensino médio em escola pública, engenharia de alimentos).

Morei no Japão por quase 10 anos e sempre quis voltar. Quanto mais tempo eu pudesse ficar no exterior, achava melhor (mulher, ensino médio em escola pública, engenharia de alimentos).

Com relação aos dados apresentados no Quadro 6, percebe-se que o indicador de capital cultural "planejamento de participação em programa de mobilidade internacional" é uma exceção comparado aos demais indicadores. A maioria das mulheres e dos homens não apresentava intenção de participar de programas antes de se tornarem cientes da existência do CsF, sugerindo que o programa foi um fator determinante na possibilidade de experiência de mobilidade internacional dos 
bolsistas CsF da Unicamp. O relato da bolsista indica a validação dessa hipótese:

[...] O CsF tinha diversas facilidades em termos de acesso $e$, especialmente a ajuda de custo. Se eu fosse por conta, não acho que teria tantas oportunidades devido custos que gerariam. Assim, ficaria menos tempo (mulher, ensino médio em escola pública, engenharia química).

Não tinha dinheiro para isso e não sabia da possibilidade de ser bancado $100 \%$ no exterior (homem, escola privada, engenharia mecânica).

Achava uma oportunidade muito distante (homem, escola pública, engenharia elétrica).

Achava que seria muito difícil e não tinha tanta confiança no meu inglês (homem, ensino médio em escola privada, engenharia elétrica).

A partir dos dados apresentados, pode-se inferir que o fato de o aluno ser oriundo de escola pública ou privada mostrou ter um impacto significativo sobre as experiências de vida $e$ expectativas de carreira entre os homens, o que acabou não ocorrendo no caso das mulheres, já que os outros indicadores de acesso ao capital cultural permaneceram praticamente inalterados entre aquelas que estudaram em escola privada ou pública. $\mathrm{O}$ capital cultural parece ter derrubado fronteiras para a inclusão das mulheres no CsF da Unicamp. Por outro lado, isso nos leva a refletir também que, para terem as mesmas oportunidades na carreira (acesso a universidade, oportunidades de intercâmbio, estágio, empregos, etc.) e alcançarem o mesmo patamar que os homens, as mulheres buscam ter acesso a oportunidades extras no decorrer da vida.

Os dados coletados via questionário também procuraram elucidar a existência de possíveis obstáculos para a participação de mulheres no CsF da Unicamp, como, por exemplo o estado civil e a existência de filhos das alunas no momento de viagem para o 
exterior. Conforme mencionado acima, mulheres casadas e com filhos tendem a ser menos móveis. Segundo dados dos questionários, nenhum dos bolsistas (homens e mulheres) relatou ter filhos no período do intercâmbio, e apenas uma mulher e dois homens relataram estar casados (formalmente ou não) no período do intercâmbio (Quadro 7). Dentre eles, somente um homem casado foi sozinho para o exterior, os demais foram acompanhados por seus cônjuges. Apesar de os dados sugerirem que o estado civil $e$ a existência de filhos dos bolsistas não foram obstáculos para o período de estadia no exterior, é possível que alunos $e$ alunas que se encontravam nessas condições nem tenham se candidatado ao programa. Para confirmar essa hipótese seria necessário obter informações entre os não participantes.

Quadro 7. Estado civil, bolsistas CsF da Unicamp, amostra selecionada por gênero

\begin{tabular}{|l|c|r|c|r|}
\hline \multicolumn{1}{|c|}{ Estado civil } & \multicolumn{2}{c|}{ Mulheres } & \multicolumn{2}{c|}{ Homens } \\
\hline No início do intercâmbio & \multicolumn{1}{c|}{$\boldsymbol{\text { \% }}$} & \multicolumn{1}{c|}{ \# } & \multicolumn{1}{c|}{$\%$} \\
\hline Casada (o) & 1 & 1,16 & 2 & 1,69 \\
\hline Solteira (o) & 60 & 69,77 & 89 & 75,42 \\
\hline Namorando & 25 & 29,07 & 27 & 22,88 \\
\hline Estado civil atual & & & & \\
\hline Casada (o) & 1 & 1,16 & 4 & 3,39 \\
\hline Solteira (o) & 54 & 62,79 & 80 & 67,80 \\
\hline Namorando & 31 & 36,05 & 34 & 28,81 \\
\hline Separada (o) & 0 & 0,00 & 0 & 0,00 \\
\hline
\end{tabular}

Fonte: elaboração própria baseada em dados coletados em questionário tipo survey.

Conforme apresentado no Quadro 7, o questionário incluiu a opção "namorando" na análise do estado civil. O objetivo da inclusão dessa opção também foi averiguar se namoros tinham se tornado obstáculos para as mulheres do CsF da Unicamp, uma vez que a idade esperada das bolsistas era entre 20 e 24 anos de 
idade. Segundo dados qualitativos coletados via questionário, o envolvimento em um relacionamento amoroso antes ou durante o período do intercâmbio se mostrou um elemento de dificuldade a ser superado por muitos bolsistas, tanto homens quanto mulheres, conforme evidenciado por alguns relatos:

Estava namorando, mas terminei no primeiro mês do intercâmbio (mulher, engenharia de alimentos).

Namorava quando fui para o intercâmbio $e$ o relacionamento acabou durante (homem, engenharia elétrica).

Me afastei dos amigos e do namorado, por eles não terem participado do intercâmbio, não conseguiam entender a dinâmica do que acontecia e acabaram por se afastar (mulher, enfermagem).

Gerou ciúmes excessivo e muitas brigas no período que fiquei fora (homem, ciência da computação).

Ainda que dificuldades nos relacionamentos amorosos tenham surgido, as expectativas futuras dos bolsistas com relação a oportunidades de intercâmbio no exterior e manutenção de um relacionamento mais estável (casamento formal ou não) sugeriram como homens e mulheres percebem possíveis barreiras para ingresso em programas de mobilidade internacional. 
Quadro 8. Repetição da experiência de mobilidade internacional se casado(a) e com filhos, bolsistas CsF da Unicamp, amostra selecionada

\begin{tabular}{|c|c|c|c|c|}
\hline Estado Civil e Existência de & \multicolumn{2}{|c|}{ Mulheres } & \multicolumn{2}{|c|}{ Homens } \\
\hline Se fosse casado(a) & \# & $\%$ & \# & $\%$ \\
\hline Não repetiria & 2 & 2,33 & 4 & 3,39 \\
\hline $\begin{array}{l}\text { Sim, independente do } \\
\text { cônjuge acompanhar }\end{array}$ & 46 & 53,49 & 65 & 55,08 \\
\hline $\begin{array}{l}\text { Sim, mas por um prazo } \\
\text { menor }\end{array}$ & 24 & 27,91 & 24 & 20,34 \\
\hline $\begin{array}{l}\text { Sim, somente com } \\
\text { acompanhamento de } \\
\text { cônjuge }\end{array}$ & 14 & 16,98 & 25 & 21,19 \\
\hline Se tivesse filhos & \# & $\%$ & \# & $\%$ \\
\hline Não repetiria & 13 & 15,12 & 24 & 20,34 \\
\hline $\begin{array}{l}\text { Sim, independente do filho } \\
\text { acompanhar }\end{array}$ & 6 & 6,98 & 15 & 12,71 \\
\hline $\begin{array}{l}\text { Sim, mas por um prazo } \\
\text { menor }\end{array}$ & 16 & 18,60 & 29 & 24,58 \\
\hline $\begin{array}{l}\text { Sim, somente com } \\
\text { acompanhamento do filho }\end{array}$ & 51 & 59,30 & 50 & 42,37 \\
\hline
\end{tabular}

Fonte: elaboração própria baseada em dados coletados em questionário tipo survey.

Os dados do Quadro 8 sugerem que mulheres não percebem o estado civil, i.e. casada, como um impedimento para suas mobilidades futuras. ${ }^{20} \mathrm{O}$ mesmo não foi identificado com relação à existência de filhos. Ainda que mais homens tenham declarado que não repetiriam a experiência se tivessem filhos quando comparados às mulheres bolsistas, uma maior proporção de homens repetiria a experiência independente do acompanhamento dos filhos. Os dados sugerem que as mulheres se tornam aparentemente menos móveis internacionalmente

\footnotetext{
${ }^{20}$ Ressalte-se que esses resultados se referem a um grupo de mulheres que ainda não vivenciaram o casamento. Dessa forma, suas percepções sobre a mobilidade futura estando em outro estado civil (casadas) devem ser relativizadas.
} 
quando há a existência de filhos (quase $60 \%$ das mulheres disseram que só repetiriam experiência se $\mathrm{o}$ filho as acompanhasse no período no exterior).

\section{Conclusões}

Este artigo investigou a inclusão de mulheres em programas internacionais de mobilidade por meio do estudo de caso de bolsistas do CsF da Unicamp para o período de 2012 a 2016 (primeiro trimestre). O estudo buscou responder às seguintes perguntas: Qual tem sido a participação de mulheres no CsF? Elas têm sido contempladas de maneira proporcional à sua presença nas universidades e carreiras privilegiadas pelo programa? Quem são as mulheres da Unicamp que vão para as áreas "masculinas" e buscam mobilidade internacional? Que características, que motivações as tornam "mulheres sem fronteiras" durante sua formação acadêmica? Elas têm as mesmas motivações que os homens, percebem as mesmas barreiras, respondem aos mesmos incentivos? São tão "livres" quanto eles para migrar temporariamente? Essas perguntas exigiram o emprego de dados secundários e coleta de dados primários no estudo, respetivamente indicadores do $\mathrm{CsF}$ para o nível do país, $e$ indicadores do CsF para a Unicamp. Os dados possibilitaram identificar um possível padrão na mobilidade internacional das mulheres da Unicamp.

Os resultados do estudo mostraram que as mulheres da Unicamp, de diferentes classes socioeconômicas, conseguiram transpor barreiras de gênero para se tornarem mulheres sem fronteiras, e um fator que parece ter sido determinante nesse processo é o acesso ao capital cultural anterior à inscrição no programa. Mulheres que receberam mais cuidados dos familiares, não somente afetivos (e.g., visita durante estágio no exterior), como financeiros (e.g., por meio de investimento em idioma estrangeiro) apresentaram maior adesão ao $\mathrm{CsF}$ na Unicamp quando comparadas aos homens. Tais resultados sugerem que para as mulheres terem as mesmas oportunidades e serem 
competitivas com os homens na carreira têm que ter acesso a inúmeras oportunidades extras durante a vida. Nesse sentido, os resultados sugerem ainda que a viagem ao exterior por meio do CsF parece ser uma continuidade na trajetória de acesso $e$ aquisição de capital cultural para as mulheres do programa. Tais resultados apresentam importantes implicações de política pública para programas de mobilidade internacional. A continuidade de programas de mobilidade como o CsF é de extrema relevância para a carreira futura de mulheres que atuam em áreas do conhecimento tradicionalmente "masculinas", e a interrupção de programas de escopo e porte do $\mathrm{CsF}$ pode acarretar em distorções nas carreiras de mulheres ao longo de suas trajetórias profissionais. Outra implicação importante de política pública se refere à importância de fomento no acesso ao capital cultural dos demais grupos da amostra de bolsistas analisada, em especial homens de escola pública. Esse grupo apresentou baixos indicadores quando comparado aos grupos de mulheres e homens com origem em escolas privadas. Uma vez que o CsF acaba favorecendo a inclusão social, o escopo do programa deve estar atrelado a outras políticas de fomento que estimulem, por exemplo, maior acesso a oportunidades $e$ vivências que possibilitem a aquisição de maior capital cultural por parte da população de baixa renda.

Os resultados apresentados neste artigo possuem alcance limitado quando à generalização dos dados. Isso porque a amostra obtida por meio de questionário tipo survey é indicativa e não exaustiva do universo de bolsistas. Ademais, algumas questões importantes precisam ser abordadas a fundo, como, por exemplo, a escolaridade dos pais dos bolsistas do $\mathrm{CsF} e$ a motivação de escolha por eles da carreira nas áreas tecnológicas. Outra limitação do estudo se refere ao fato de os resultados obtidos estarem relacionados a um perfil específico de universidade - a Unicamp é uma universidade com longa tradição em pesquisa $e$ a maioria do alunado é oriunda de escola privada. Um estudo sobre a inclusão de mulheres em programas de mobilidade internacional em uma universidade de menor porte, menor tradição de pesquisa e com 
perfil de alunado diferente do da Unicamp pode potencialmente trazer resultados distintos daqueles encontrados no aqui apresentado, e, assim, torna-se uma possibilidade de estudos futuros.

\section{Referências bibliográficas}

ACKERS, L. Managing relationships in peripatetic careers: Scientific mobility in the european union. Women's Studies International Quarterly, 27, 2004, pp.189-201.

AdAmuti-Trache, M.; ANDRES, L. Embarking on and Persisting in Scientific Fields of Study: Cultural capital, gender, and curriculum along the science pipeline. International Journal of Science Education, 30(12), 2008, pp.1557-1584.

BALBACHEVSKY, E. Brazil's high education responses to the global challenges of the 21st century. Thinking Brazil, n.23, 2006. Disponivel em

http://s3.amazonaws.com/academia.edu.documents/33275050/Thinki ngBrazil_23.pdf?AWSAccessKeyId=AKIAJ56TQJRTWSMTNPEA\&Ex pires $=1 \overline{4} 76733777 \&$ Signature $=\mathrm{R} 1 \mathrm{VMYmt0} \% 2 \mathrm{BuNNfxiY} 8 \mathrm{wCUxpH} 1$ ABk\%3D\&response-contentdisposition=inline\%3B\%20filename\%3DBrazil_s_High_Education_Re sponses_to_the.pdf >

BALBACHEVSKY, E.; MARQUES, F. "Fuga de cerebros" en Brasil: los costos públicos del errado entendimiento de una realidad acadêmica. In: AuPETIT, S. D.; GÉRARD, E. (eds.) Fuga de cerebros, movilidad académica, redes científicas: perspectivas latinoamericanas. México, D.F.; CINVESTAV, 2009, pp.161-173.

BLICKENSTAFF, J.C. Women and science careers: Leaky pipeline or gender filter? Gender and Education, 17(4), 2005, pp.369-386.

Bourdieu, P. O poder simbólico. $2^{\text {a }}$ ed. Rio de Janeiro, Bertrand Brasil, 1998 [Tradução Fernando Tomaz].

BOZEMAN, B.; CORLEY, E. Scientists' collaboration strategies: implications for scientific and technical human capital. Research Policy, 33(4), 2004, pp.599-616. 
BroOKS, R; WATERS, J. Student Mobilities, Migration and the Internationalization of Higher Education. Palgrave MacMillan, Basingstoke, 2011.

CASTRO, C. M. Brain drain in Latin America: myth and reality, 2007 [http://www.claudiomouracastro.com.br/upload/Brain\%20drain\%20in \%20Latin\%20America\%20myth\%20and\%20reality.pdf - acesso em: 22 jul 2011].

CLARK, W.A.V.; WITHERS, S. D. Disentangling the interaction of migration, mobility, and labor-force partic- ipation. Environment and Planning A, 34, 2002, pp.923-945.

Etzkowitz, H.; Kemelgor, C.; Uzzi, B. Athena unbound: The advancement of women in science and technology. Cambridge, UK, Cambridge University Press, 2000.

FAusto-Sterling, A. Duelismos em duelo. cadernos pagu (17/18), Campinas, Núcleo de Estudos de Gênero-Pagu/Unicamp, 2001/02, pp.9-79.

GONZÁLEZ, A. M.; VERGÉS, N. International mobility of women in science and technology careers: shaping plans for personal and professional purposes. Gender, Place \& Culture, 20 (5), 2013, pp.613-629.

HANSON, S.L. Lost talent. Women in the sciences. Philadelphia, Temple University Press, 1996.

HIRATA, Helena; GuimARÃES, Nadya Araujo. Cuidado e Cuidadoras - As Várias Faces do Trabalho do Care. São Paulo: Editora Atlas S.A., 2012.

INEP. Instituto Nacional de Estudos e Pesquisas Educacionais Anísio Teixeira (INEP). Resumo Técnico Censo da Educação Superior, 2013. Diretoria de Estatísticas Educacionais (DEED). Ministério da Educação, 2013.

JANnuzZi, P. M. Considerações sobre o uso, mau uso e abuso dos indicadores sociais na formulação e avaliação de políticas públicas municipais. Revista de Administração Pública, 36(1), jan./fev. 2002, pp.51-72.

KELLER, E F. Reflexiones sobre género y ciencia. Edicions Alfons El Magnànim, Valencia, 1991. 
LiMA, M.C. As mulheres na ciência da computação. Revista Estudos Feministas, 21(3), 2013, pp.793-816.

LOMBARDI, M. R. Engenheiras Brasileiras: Inserção e Limites de Gênero no Campo Profissional. Cadernos de Pesquisa, vol. 36, $\mathrm{n}^{\circ}$ 127, jan./abr. 2006.

MCCALL, Leslie. The complexity of intersectionality. Signs: Journal of Women in Culture and Society, vol. 30, n 3, 2005, pp.1771-1800.

MAHROUM, S. Highly skilled globetrotters: mapping the international migration of human capital. R\&D Management, vol. 30, ${ }^{\circ} 1,2000$, pp.23-32.

SCHWARTZMAN, S. Nacionalismo versus internacionalismo en las políticas de formación de recursos humanos de alto nível. In: AUPETIT, S. D.; GÉRARD, E. (eds.). Fuga de cerebros, movilidad académica, redes científicas: perspectivas latinoamericanas. México, D.F., CINVESTAV, 2009, pp.63-73.

TEICHLER, U.; MAIWORM, F. The ERASMUS Experience. Major findings of the ERASMUS Evaluation Research Project. Brussels7 CEC, 1997.

THE ROYAL SOCIETY. Knowledge, networks and nations: global scientific collaboration in the 21st century. Londres, The Royal Society, 2011.

VELHO, L. Formação de doutores no país e no exterior: estratégias alternativas ou complementares? Dados, vol .44, n³, 2001, pp.607631.

Disponível

em

$<$

http://www.scielo.br/scielo.php?script=sci_arttext\&pid=S0011$52582001000300005>$

VELHO, L. Formação de recursos humanos qualificados e sistema de inovação. In: AlBORNOZ, M.; VOGT, C.; AlfarAZ, C. (eds.). Indicadores de ciencia y tecnologia en Iberoamerica - agenda 2008, Buenos Aires, RICYT. pp. 139-148.

VELHO, L. INTERNACIONALIZAÇÃO DA CIÊNCIA: ACASO OU NECESSIDADE? JORNAL DA UNICAMP, CAMPINAS, ANO 25, N.505, 11 SET. 2011. DISPONIVEL EM: < HTTP://WWW.UNICAMP.BR/UNICAMP/UNICAMP_HOJE/JU/SETEMBRO2011/JU 505_PAG2.PHP >. 
Velloso, J. (Org.). Formação no país ou no exterior? Doutores na pósgraduação de excelência. Um estudo na bioquímica, engenharia elétrica, física e química no país. Brasília, Capes, 2002.

WAGNER, C. S. International collaboration in science: a new dynamic for knowledge creation. Tese (Doutorado em Ciência e Tecnologia), University of Amsterdam, 2004.

WoOlLEY, R. et al. Mobility matters - research training and network building in science. Comparative Technology Transfer and Society, 6(3), 2008, pp.159-186. 